\title{
The Cameron-Martin Theorem for (p-)Slepian processes
}

\author{
Wolfgang Bischoff* and Andreas Gegg \\ Faculty of Mathematics and Geography, \\ Catholic University of Eichstätt-Ingolstadt,
}

\begin{abstract}
We show a Cameron-Martin theorem for Slepian processes $W_{t}:=$ $\frac{1}{\sqrt{p}}\left(B_{t}-B_{t-p}\right), t \in[p, 1]$, where $p \geq \frac{1}{2}$ and $B_{s}$ is Brownian motion. More exactly, we determine the class of functions $F$ for which a density of $F(t)+W_{t}$ with respect to $W_{t}$ exists. Moreover, we prove an explicit formula for this density. p-Slepian processes are closely related to Slepian processes. p-Slepian processes play a prominent role among others in scan statistics and in testing for parameter constancy when data are taken from a moving window.
\end{abstract}

Keywords: Cameron-Martin theorem, (p-)Slepian process, Radon-Nikodym derivative

subject classification: $60 \mathrm{G} 15,60 \mathrm{H} 99$

\section{Introduction}

A Cameron-Martin theorem for a stochastic process is one of the most useful tools to solve problems the process is involved in. Let a stochastic process $X_{[a, b]}=\left(X_{t}\right)_{t \in[a, b]}$ with paths in $C[a, b]$, the set of real-valued and continuous functions on $[a, b] \subseteq \mathbb{R}$, and a deterministic function $F \in C[a, b]$ be given. Let $\mathbb{P}^{X_{[a, b]}}$ and $\mathbb{P}^{(X+F)_{[a, b]}}$ denote the distribution of $X_{[a, b]}$ and of $\left(X_{t}+F(t)\right)_{t \in[a, b]}$ on $C[a, b]$, respectively. Then a Cameron-Martin theorem gives conditions on $F$ under which a density $\frac{d\left(\mathbb{P}^{X_{t}+F(t)}\right)}{d \mathbb{P}^{X}}$ exists and, additionally, it gives an explicit formula for this density.

The first and best known result of this type is by 6. They proved it for the standard Brownian motion $B_{[0,1]}:=\left(B_{t}\right)_{t \in[0,1]}$ with continuous paths. This Cameron-Martin theorem can be used, for example, to calculate optimal tests, see 4, and to estimate boundary crossing probabilities, see 5. Therefore, such a result of Cameron-Martin type is also of great interest for other stochastic processes. The following theorem, see Lifshits [12, Theorem 5.1], can be used as basis to get results of Cameron-Martin type for centered Gaussian processes. To this end, let $L^{2}(C[a, b], P)$ be the set of all real-valued and square-integrable

\footnotetext{
*corresponding author: Wolfgang Bischoff, Faculty of Mathematics and Geography, D85071 Eichstätt, Germany; E-Mail: wolfgang.bischoff@ku-eichstaett.de
} 
functions on $C[a, b]$ with respect to a measure $P$ defined on the Borel- $\sigma$-Algebra of $C[a, b]$.

\section{Theorem 1.1 (An abstract Cameron-Martin theorem)}

Let $X_{[a, b]}:=\left(X_{t}\right)_{t \in[a, b]}$ be a centered Gaussian process with paths in $C[a, b]$, let $\mathcal{H}=\mathcal{H}_{X_{[a, b]}} \subseteq C[a, b]$ be the kernel of $\mathbb{P}^{X_{[a, b]}}$ and $\|\cdot\|_{\mathcal{H}}$ its inherent norm. Then,

$$
\mathbb{P}^{(X+h)_{[a, b]}} \text { is absolutely continuous with respect to } \mathbb{P}_{[a, b]}^{X_{[a,}} \Longleftrightarrow h \in \mathcal{H} .
$$

If $h \in \mathcal{H}$, then

$$
\frac{d \mathbb{P}^{(X+h)_{[a, b]}}}{d \mathbb{P}^{X_{[a, b]}}}(g)=\exp \left(-\frac{1}{2}\|h\|_{\mathcal{H}}^{2}+z(g)\right) \quad \text { for } \mathbb{P}^{X_{[a, b]} \text { almost all } g \in C([a, b]),}
$$

where $z=z_{X_{[a, b]}} \in L^{2}\left(C[a, b], \mathbb{P}^{X_{[a, b]}}\right)$ is a linear functional fulfilling the equation

$$
\mathbf{E}\left(X_{t} \cdot z_{X_{[a, b]}}\right)=h(t), \quad t \in[a, b] .
$$

Hence, the kernel $\mathcal{H}=\mathcal{H}_{X_{[a, b]}}$ of the stochastic process $X_{[a, b]}$ together with its inherent norm $\|\cdot\|_{\mathcal{H}}$ and the functional $z_{X_{[a, b]}}$ must be determined to obtain an applicable result.

In the following, we use the notation stated in the next definition.

\section{Definition 1.2}

Let $X_{[a, b]}:=\left(X_{t}\right)_{t \in[a, b]}$ be a centered Gaussian process with paths in $C[a, b]$ and let $\mathcal{H}=\mathcal{H}_{X_{[a, b]}} \subseteq C[a, b]$ be the kernel of $\mathbb{P}^{X_{[a, b]}}$. Then, we say that a function

$$
z=z_{X_{[a, b]}}: \mathcal{H}_{X_{[a, b]}} \times C[a, b] \rightarrow \mathbb{R}
$$

fulfills condition $A$ for $X_{[a, b]}$, if and only if for all $h \in \mathcal{H}_{X_{[a, b]}}$ the function $z(h, \cdot) \in L^{2}\left(C[a, b], \mathbb{P}^{\left.X_{[a, b]}\right)}\right.$ is a linear functional fulfilling

$$
\mathbf{E}\left(X_{t} \cdot z\left(h, X_{[a, b]}\right)\right)=h(t), \quad t \in[a, b] .
$$

For the Brownian motion, we have the following result by 6 . To this end, let $\lambda$ denote the Lebesgue measure on $\mathbb{R}$ and let $L^{2}[a, b]:=L^{2}([a, b], \lambda)$ be the square-integrable functions on $[a, b]$ with respect to $\lambda$.

\section{Theorem 1.3}

The kernel of $B_{[0,1]}$ is given by

$$
\mathcal{H}_{B_{[0,1]}}:=\left\{s_{f} \in C[0,1] \mid f \in L^{2}[0,1]\right\},
$$

where

$$
s_{f}(t):=\int_{[0, t]} f d \lambda, t \in[0,1], \text { for } \lambda \text {-integrable } f:[0,1] \rightarrow \mathbb{R} .
$$

It is furnished with the norm

$$
\left\|s_{f}\right\|_{\mathcal{H}_{B_{[0,1]}}}:=\|f\|_{L^{2}[0,1]}, \quad f \in L^{2}[0,1] .
$$


A function $z:=z_{B_{[0,1]}}$ fulfilling condition $A$ for $B_{[0,1]}$ is given by the Wiener integral, see, for instance, [14],

$$
z: \mathcal{H}_{B_{[0,1]}} \times C[0,1] \rightarrow \mathbb{R}, \quad\left(s_{f}, b\right) \rightarrow \int_{0}^{1} f d b .
$$

Slepian and p-Slepian processes are defined and their close relations are discussed in the next section. In Sect. 3, results of Cameron-Martin type are established for p-Slepian processes. Several proofs are postponed to an appendix.

\section{Slepian and p-Slepian processes}

The Slepian process $\mathrm{X}$ is the centered stationary Gaussian process with covariance function

$$
C_{X}\left(s^{\prime}, s^{\prime}+u\right)=(1-u)^{+} \quad, 0 \leq s^{\prime} \leq s^{\prime}+u,
$$

where $t^{+}=\max (0, t), t \in \mathbb{R}$. This process was introduced and studied in 18 and later in $[16,17]$. Afterward, it was handled in numerous theoretical and applied probabilistic models; see, e.g., [1-3,9-11,13,15].

Let us consider the Slepian process on time intervals $[1, b]$, where $1<b$ is any fixed constant. Let $\nabla_{p}, p>0$, be the backward difference operator with $\operatorname{lag} p$, i.e., $\nabla_{p} F(t)=F(t)-F(t-p), t \in \mathbb{R}$, for functions $F: \mathbb{R} \rightarrow \mathbb{R}$. Note that the Slepian process coincides in distribution with

$$
\left(\nabla_{1} B\right)_{[1, b]}:=\left(\nabla_{1} B_{t}\right)_{t \in[1, b]}=\left(B_{t}-B_{t-1}\right)_{t \in[1, b]},
$$

where $B_{t}$ is the standard Brownian motion with continuous paths in $[0, b]$. In Cressie [8, pp. 834], a slightly different expression of Slepian processes appeared in connection with scan statistics. They were considered the processes

$$
\frac{1}{\sqrt{p}}\left(\nabla_{p} B\right)_{[p, 1]}:=\frac{1}{\sqrt{p}}\left(\nabla_{p} B_{t}\right)_{t \in[p, 1]}=\frac{1}{\sqrt{p}}\left(B_{t}-B_{t-p}\right)_{t \in[p, 1]}
$$

for any fixed constant $p \in(0,1)$. We call this process $p$-Slepian process. Another application of p-Slepian processes is given by 7 . They consider moving sums of recursive residuals which are taken from windows of length $p$. By letting the number of residuals to $\infty$, they get the p-Slepian process $\frac{1}{\sqrt{p}}\left(\nabla_{p} B\right)_{[p, 1]}$.

By a suitable scaling in time the Slepian process $\left(\nabla_{1} B\right)_{[1, b]}, b \in(1, \infty)$, can be transferred to the p-Slepian process $\frac{1}{\sqrt{p}}\left(\nabla_{p} B\right)_{[p, 1]}, p \in(0,1)$, by putting $p=\frac{1}{b}$. More exactly it holds $\left(\nabla_{1} B_{u}\right)_{u \in[1, b]}=\sqrt{b}\left(\nabla_{\frac{1}{b}} B_{\frac{u}{b}}\right)_{u \in[1, b]}$ in distribution. The covariance function of a p-Slepian process is given by

$$
C_{\frac{1}{\sqrt{p}}\left(\nabla_{p} B\right)_{[p, 1]}}(s, s+t)=\left(1-\frac{t}{p}\right)^{+}, p \leq s \leq s+t \leq 1 .
$$




\section{Cameron-Martin Theorem for p-Slepian pro- cesses}

The function $\frac{1}{\sqrt{p}} \nabla_{p}, 0<p \leq 1$, is linear. Hence, by [12] proposition 4.1, the kernel $\mathcal{H}_{p-\mathrm{Sl}}$ of the p-Slepian process $\frac{1}{\sqrt{p}}\left(\nabla_{p} B\right)_{[p, 1]}$ is given by $\mathcal{H}_{p-S l}=$ $\frac{1}{\sqrt{p}} \nabla_{p}\left(\mathcal{H}_{B_{[0,1]}}\right)=\left\{\frac{1}{\sqrt{p}} \nabla_{p} h \mid h \in \mathcal{H}_{B_{[0,1]}}\right\}$. By some calculations we get

$$
\begin{aligned}
\mathcal{H}_{p-\mathrm{S} 1} & =\left\{\frac{1}{\sqrt{p}} \nabla_{p} s_{f}:[p, 1] \rightarrow \mathbb{R} \mid f \in L^{2}[0,1]\right\} \\
& =\left\{c+s_{g}:[p, 1] \rightarrow \mathbb{R} \mid c \in \mathbb{R}, g \in L^{2}[p, 1]\right\} .
\end{aligned}
$$

More exactly, we have for $f \in L^{2}[0,1]$

$$
\frac{1}{\sqrt{p}} \nabla_{p} s_{f}(t)=\frac{1}{\sqrt{p}} s_{f}(p)+s_{\frac{1}{\sqrt{p}}} \nabla_{p} f(t), t \in[p, 1] .
$$

It is much more complicated to determine the inherent norm of $\mathcal{H}_{p-\mathrm{Sl}}$ since $\nabla_{p}$ is not injective. For the following, the information of $\nabla_{p} s_{f}$ is important. For $f, g \in L^{2}[0,1]$, it holds true

$$
\begin{aligned}
& \forall t \in[p, 1]: \nabla_{p} s_{f}(t)=s_{f}(p)+\int_{p}^{t} f(s)-f(s-p) d s=\nabla_{p} s_{g}(t) \Longleftrightarrow \\
& f(t)-f(t-p)=g(t)-g(t-p) \lambda-a . s . \text { for all } t \in[p, 1], \quad s_{f}(p)=s_{g}(p) .
\end{aligned}
$$

We prove the following result in the appendix.

Lemma 3.1 Let $1 / 2 \leq p \leq 1$. Then the kernel $\mathcal{H}_{p-S l}$ is furnished with its inherent norm

$$
\begin{aligned}
& \left\|\frac{1}{\sqrt{p}} \nabla_{p} s_{f}\right\|_{p-S l}^{2}=\frac{1}{p} \inf _{g \in L^{2}[0,1]: \nabla_{p} s_{f}=\nabla_{p} s_{g}}\|g\|_{L^{2}[0,1]}^{2} \\
& =\frac{1}{2 p(3 p-1)}\left(2 s_{f}(p)+\delta(1-p)\right)^{2}+\frac{1}{2 p}\left\|(f(t)-f(t-p))_{t \in[p, 1]}\right\|_{L^{2}[p, 1]}^{2},
\end{aligned}
$$

where $\delta=\frac{1}{1-p}\left(s_{f}(1)-s_{f}(p)-s_{f}(1-p)\right)$. This minimum is attained at the function

$$
\begin{aligned}
f^{*}(t)= & \left(\frac{1}{3 p-1}\left(s_{f}(p)+\frac{1-p}{2} \delta\right)+\frac{1}{2}(-f(t+p)+f(t))\right) \mathbf{1}_{[0,1-p]}(t) \\
& +\frac{1}{2 p-1}\left(s_{f}(p)-\frac{1-p}{3 p-1}\left(s_{f}(p)-(2 p-1) \delta\right)\right) \mathbf{1}_{(1-p, p)}(t) \\
& +\left(\frac{1}{3 p-1}\left(s_{f}(p)+\frac{1-p}{2} \delta\right)+\frac{1}{2}(f(t+p)-f(t))\right) \mathbf{1}_{[p, 1]}(t), t \in[0,1] .
\end{aligned}
$$

Finally, we need the function $z:=z_{\frac{1}{\sqrt{p}}\left(\nabla_{p} B\right)_{[p, 1]}}$ fulfilling condition A for the pSlepian process to be in the position to state a result of Cameron-Martin type. 
Lemma 3.2 Let $1 / 2 \leq p \leq 1$. The function $z:=z_{\frac{1}{\sqrt{p}}\left(\nabla_{p} B\right)_{[p, 1]}}$ defined by

$$
\begin{gathered}
z: \mathcal{H}_{\frac{1}{\sqrt{p}}\left(\nabla_{p} B\right)_{[0,1]}} \times C[p, 1] \rightarrow \mathbb{R}, \quad\left(\frac{1}{\sqrt{p}} s_{f}(p)+s_{\frac{1}{\sqrt{p}} \nabla_{p} f}, \frac{1}{\sqrt{p}} \nabla_{p} b\right) \mapsto \\
\frac{3 s_{f}(p)+s_{\nabla_{p} f}(1)}{2(3 p-1)}\left(\nabla_{p} b(p)+\nabla_{p} b(1)\right)+\frac{1}{2} \int_{p}^{1} \nabla_{p} f(s) d\left(\nabla_{p} b(s)\right)
\end{gathered}
$$

fulfills condition $A$ for $\frac{1}{\sqrt{p}}\left(\nabla_{p} B\right)_{[p, 1]}$. Note that the integral

$$
\int_{p}^{1} \nabla_{p} f(s) d\left(\nabla_{p} B(s)\right)=\int_{p}^{1} \nabla_{p} f(s) d B(s)-\int_{p}^{1} \nabla_{p} f(s) d B(s-p)
$$

has to be understood as Wiener Integral.

The proof is given in the appendix. By the above lemmas, we obtain the following result.

\section{Theorem 3.3 (Cameron-Martin theorem for p-Slepian processes)}

Let $1 / 2 \leq p \leq 1$. It holds true

$$
\begin{gathered}
\mathbb{P}^{\left(\frac{1}{\sqrt{p}} \nabla_{p} B+h\right)_{[p, 1]}} \text { is absolutely continuous with respect to } \mathbb{P}^{\left(\frac{1}{\sqrt{p}} \nabla_{p} B\right)_{[p, 1]}} \\
\Longleftrightarrow h \in \mathcal{H}_{p-S l} .
\end{gathered}
$$

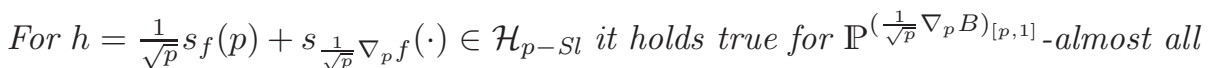
$\frac{1}{\sqrt{p}} \nabla_{p} b \in C[p, 1]:$

$$
\begin{aligned}
& \frac{d \mathbb{P}^{\left(\frac{1}{\sqrt{p}} \nabla_{p} B+h\right)_{[p, 1]}}}{d \mathbb{P}^{\left(\frac{1}{\sqrt{p}} \nabla_{p} B\right)_{[p, 1]}}}\left(\frac{1}{\sqrt{p}} \nabla_{p} b\right) \\
& =\exp \left(-\frac{1}{4 p}\left(\frac{\left(2 s_{f}(p)+\delta(1-p)\right)^{2}}{3 p-1}+\|(f(\cdot)-f(\cdot-p))\|_{L^{2}[p, 1]}^{2}\right)\right. \\
& \left.\quad+\frac{3 s_{f}(p)+s_{\nabla_{p} f}(1)}{2(3 p-1)}\left(\nabla_{p} b(p)+\nabla_{p} b(1)\right)+\frac{1}{2} \int_{p}^{1} \nabla_{p} f(s) d\left(\nabla_{p} b(s)\right)\right),
\end{aligned}
$$

where $\delta=\frac{1}{1-p}\left(s_{f}(1)-s_{f}(p)-s_{f}(1-p)\right)$.

\section{Appendix}

Proof of Lemma 3.1 Let $\frac{1}{\sqrt{p}} \nabla_{p} s_{f} \in \mathcal{H}_{p-\mathrm{Sl}}, f \in L^{2}[0,1]$, be arbitrary and let $1 / 2 \leq p \leq 1$. There are several places where the case $p=1 / 2$ must be considered separately. We do not do this since the specifications for $p=1 / 2$ are simpler than the following considerations for $1 / 2<p \leq 1$. By proposition 4.1 of [12], we get

$$
\begin{aligned}
\left\|\frac{1}{\sqrt{p}} \nabla_{p} s_{f}\right\|_{p-S l} & =\frac{1}{\sqrt{p}} \inf _{g \in L^{2}[0,1]: \nabla_{p} s_{f}=\nabla_{p} s_{g}}\left\|s_{g}\right\|_{\mathcal{H}_{B_{[0,1]}}} \\
& =\frac{1}{\sqrt{p}} \inf _{g \in L^{2}[0,1]: \nabla_{p} s_{f}=\nabla_{p} s_{g}}\|g\|_{L^{2}[0,1]},
\end{aligned}
$$


where the second equality follows by Theorem 1.3 . Each function $f \in L^{2}[0,1]$ can be written $\lambda-a . s$. in the form

$$
f(t)=\alpha \mathbf{1}_{[0,1-p]}(t)+a(t)+\beta \mathbf{1}_{(1-p, p)}(t)+b(t)+\gamma \mathbf{1}_{[p, 1]}(t)+c(t), t \in[0,1],
$$

where

$$
\begin{aligned}
& \alpha=\frac{1}{1-p} s_{f}(1-p), a(t)=\mathbf{1}_{[0,1-p]}(t)(f(t)-\alpha), \\
& \beta=\frac{1}{2 p-1}\left(s_{f}(p)-s_{f}(1-p)\right), b(t)=\mathbf{1}_{(1-p, p)}(t)(f(t)-\beta), \\
& \gamma=\frac{1}{1-p}\left(s_{f}(1)-s_{f}(p)\right), c(t)=\mathbf{1}_{[p, 1]}(t)(f(t)-\gamma) .
\end{aligned}
$$

Note that the six summands in (7) build orthogonal functions in $L^{2}[0,1]$. In the following, we use the consequence of this representation at several places without citing it explicitly. Furthermore, we have

$$
s_{f}(p)=\alpha(1-p)+\beta(2 p-1), f(t)-f(t-p)=\gamma-\alpha+c(t)-a(t), t \in[p, 1] .
$$

Next, we consider $g \in L^{2}[0,1]$ of the specific form

$$
g(t)=\alpha \mathbf{1}_{[0,1-p]}(t)+\beta \mathbf{1}_{(1-p, p)}(t)+(\alpha+\delta) \mathbf{1}_{[p, 1]}(t), t \in[0,1], \alpha, \beta, \delta \in \mathbb{R} .
$$

Hence,

$$
s_{g}(p)=\alpha(1-p)+\beta(2 p-1) \Leftrightarrow \beta=\frac{1}{2 p-1}\left(s_{g}(p)-\alpha(1-p)\right)
$$

and

$$
g(t)-g(t-p)=\delta, t \in[p, 1]
$$

Thus, $\nabla_{p}(g)(t)=s_{g}(p)+\delta t, t \in[p, 1]$. Let $g_{0}(t)=\alpha_{0} \mathbf{1}_{[0,1-p]}(t)+\beta_{0} \mathbf{1}_{(1-p, p)}(t)+$ $\left(\alpha_{0}+\delta_{0}\right) \mathbf{1}_{[p, 1]}(t) \in L^{2}[0,1]$ be fixed and let $g \in L^{2}[0,1]$ with $\nabla_{p}(g)(t)=$ $\nabla_{p}\left(g_{0}\right)(t)=s_{g_{0}}(p)+\delta_{0} t, t \in[p, 1]$. Then,

$$
g(t)=\alpha \mathbf{1}_{[0,1-p]}(t)+\frac{1}{2 p-1}\left(s_{g_{0}}(p)-\alpha(1-p)\right) \mathbf{1}_{(1-p, p)}(t)+\left(\alpha+\delta_{0}\right) \mathbf{1}_{[p, 1]}(t)
$$

and the square of its norm is given by

$$
\|g\|^{2}=\frac{1-p}{2 p-1}\left(\alpha^{2}(3 p-1)-2 \alpha\left(s_{g_{0}}(p)-(2 p-1) \delta_{0}\right)\right)+\frac{s_{g_{0}}(p)^{2}}{2 p-1}+\delta_{0}^{2}(1-p) .
$$

This norm is minimal if and only if

$$
\alpha=\frac{1}{3 p-1}\left(s_{g_{0}}(p)-(2 p-1) \delta_{0}\right) .
$$

Hence, we obtain after some calculation

$$
\left\|\nabla_{p} s_{g_{0}}\right\|_{p-S l}^{2}=\frac{1}{3 p-1}\left(2 s_{g_{0}}(p)^{2}+2(1-p) s_{g_{0}}(p) \delta_{0}+\delta_{0}^{2}(1-p) p\right) .
$$

Next, we consider a general function

$$
f=\alpha \mathbf{1}_{[0,1-p]}(t)+a(t)+\beta \mathbf{1}_{(1-p, p)}(t)+b(t)+(\alpha+\delta) \mathbf{1}_{[p, 1]}(t)+c(t) \in L^{2}[0,1] .
$$


By the above considerations we obtain

$$
\begin{aligned}
\left\|\nabla_{p} s_{f}\right\|_{p-S l}^{2}= & \frac{1}{3 p-1}\left(2 s_{f}(p)^{2}+2(1-p) s_{f}(p) \delta+\delta^{2}(1-p) p\right) \\
& +\min \left(\|a(t)\|^{2}+\|b(t)\|^{2}+\|c(t)\|^{2}\right)
\end{aligned}
$$

where the minimum is taken over all $a:[0,1-p] \rightarrow \mathbb{R}, b:(1-p, p) \rightarrow \mathbb{R}, c:$ $[p, 1] \rightarrow \mathbb{R}$ with $s_{a}(1-p)=s_{b}(2 p-1)=s_{c}(1)=0$ and $c(t)-a(t-p)=$ $f(t)-f(t-p)-\delta$. It holds true for $t \in[p, 1]$ fixed

$$
\begin{aligned}
\min |a(t-p)|^{2}+|c(t)|^{2} & =\left|\frac{1}{2}(-c(t)+a(t-p))\right|^{2}+\left|\frac{1}{2}(c(t)-a(t-p))\right|^{2} \\
& =\frac{1}{2}|(c(t)-a(t-p))|^{2}=\frac{1}{2}|f(t)-f(t-p)-\delta|^{2},
\end{aligned}
$$

where the minimum is taken over all $a(t-p), c(t) \in \mathbb{R}$ with $c(t)-a(t-p)=$ $f(t)-f(t-p)-\delta$. Hence,

$$
\begin{aligned}
\left\|\nabla_{p} s_{f}\right\|_{p-S l}^{2}= & \frac{1}{3 p-1}\left(2 s_{f}(p)^{2}+2(1-p) s_{f}(p) \delta+\delta^{2}(1-p) p\right) \\
& +\min \|a(t)\|_{L^{2}[0,1]}^{2}+\|c(t)\|_{L^{2}[0,1]}^{2} \cdot \\
= & \frac{1}{3 p-1}\left(2 s_{f}(p)^{2}+2(1-p) s_{f}(p) \delta+\delta^{2}(1-p) p\right) \\
& +\frac{1}{2}\left\|(f(t)-f(t-p)-\delta)_{t \in[p, 1]}\right\|_{L^{2}[p, 1]}^{2} \\
= & \frac{1}{3 p-1}\left(2 s_{f}(p)^{2}+2(1-p) s_{f}(p) \delta+\delta^{2}(1-p) p\right) \\
& +\frac{1}{2}\left(\left\|(f(t)-f(t-p))_{t \in[p, 1]}\right\|_{L^{2}[p, 1]}^{2}-(1-p) \delta^{2}\right) \\
= & \frac{1}{2(3 p-1)}\left(2 s_{f}(p)+\delta(1-p)\right)^{2} \\
& +\frac{1}{2}\left\|(f(t)-f(t-p))_{t \in[p, 1]}\right\|_{L^{2}[p, 1]}^{2} .
\end{aligned}
$$

This minimum is obtained at the function

$$
\begin{aligned}
f^{*}(t)= & \left(\frac{1}{3 p-1}\left(s_{f}(p)+\frac{1-p}{2} \delta\right)+\frac{1}{2}(-f(t+p)+f(t))\right) \mathbf{1}_{[0,1-p]}(t) \\
& +\frac{1}{2 p-1}\left(s_{f}(p)-\frac{1-p}{3 p-1}\left(s_{f}(p)-(2 p-1) \delta\right)\right) \mathbf{1}_{(1-p, p)}(t) \\
& +\left(\frac{1}{3 p-1}\left(s_{f}(p)+\frac{1-p}{2} \delta\right)+\frac{1}{2}(f(t+p)-f(t))\right) \mathbf{1}_{[p, 1]}(t), t \in[0,1] .
\end{aligned}
$$

Proof of Lemma 3.2 We have to prove Eq. (1) for the p-Slepian-process. To this end, let $p \leq t \leq 1, f \in L^{2}[0,1]$ and $\left(\nabla_{p} s_{f}\right)(\cdot)=s_{f}(p)+s_{\nabla_{p} f}(\cdot) \in \mathcal{H}_{p-\text { Sl }}$. It 
holds true for $t \in[p, 1]$ :

$$
\begin{aligned}
& \mathbf{E}\left(\frac{1}{\sqrt{p}}\left(\nabla_{p} B\right)_{[p, 1]}(t) \cdot \frac{3 s_{f}(p)+s_{\nabla_{p} f}(1)}{2(3 p-1)}\left(\left(\nabla_{p} B\right)_{[p, 1]}(p)+\left(\nabla_{p} B\right)_{[p, 1]}(1)\right)\right) \\
& \left.\quad+\mathbf{E}\left(\frac{1}{\sqrt{p}}\left(\nabla_{p} B\right)_{[p, 1]}(t) \cdot \frac{1}{2} \int_{p}^{1} \nabla_{p} f(s) d\left(\nabla_{p} B\right)_{[p, 1]}(s)\right)\right) \\
& =\frac{3 s_{f}(p)+s_{\nabla_{p} f}(1)}{2 \sqrt{p}(3 p-1)} \mathbf{E}\left(\left(B_{[0,1]}(t)-B_{[0,1]}(t-p)\right)\left(B_{[0,1]}(p)+B_{[0,1]}(1)-B_{[0,1]}(1-p)\right)\right) \\
& \quad+\frac{1}{2 \sqrt{p}} \mathbf{E}\left(\left(B_{[0,1]}(t)-B_{[0,1]}(t-p)\right) \int_{p}^{1} \nabla_{p} f(s) d\left(B_{[0,1]}(s)-B_{[0,1]}(s-p)\right)\right) \\
& =\frac{3 s_{f}(p)+s_{\nabla_{p} f}(1)}{2 \sqrt{p}}-\frac{1}{2 \sqrt{p}}\left(s_{\nabla_{p} f}(1)-s_{\nabla_{p} f}(p)\right)=\frac{1}{\sqrt{p}} s_{f}(p)+s_{\frac{1}{\sqrt{p}} \nabla_{p} f}(t) .
\end{aligned}
$$

\section{References}

[1] Abrahams, J. (1984). Ramp crossings for Slepian's process. IEEE Trans. Inform. Theory, 30 No. 3, 574-575.

[2] AdLer (1990). An introduction to continuity, extrema, and related topics for general Gaussian processes, Hayward, Calif., Institute of Mathematical Statistics, Lecture notes, monograph series 12.

[3] Bar-David (1975). Radon-Nikodym Derivatives, Passages and Maxima for a Gaussian Process with Particular Covariance and Mean. Journal of Applied Probability, 12, 724-733.

[4] Bischoff, W. and Miller, F. (2000). Asymptotically optimal tests and optimal designs for testing the mean in regression models with applications to change-point problems. Ann. Inst. Stat. Math., 52, 658-679.

[5] Bischoff, W. and Hashorva, E. (2005). A lower bound for boundary crossing probabilities of Brownian bridge with trend. Stat. Probab. Lett., 74, 265-271.

[6] Cameron, R. H. and Martin, W. T. (1944). Transformations of Wiener Integrals Under Translations. Annals of Mathematics, 45, 386-396.

[7] Chu, C., HorniK, K. and Kaun, C. (1995). MOSUM tests for parameter constancy. Biometrika, 82, 603-617.

[8] Cressie, N. (1980). The Asymptotic Distribution of the Scan Statistic under Uniformity. The Annals of Probability, 8, 828-840.

[9] Gao, F. and Li, W. (2007). Small ball probabilities for the Slepian Gaussian fields. Trans. Am. Math. Soc., 359 No. 3, 1339-1350.

[10] GegG, A. (2013). Moving Windows zum Auffinden von Change-Points. Doktor Thesis (in German), Catholic University of Eichstätt-Ingolstadt. 
[11] Jamison (1970). Reciprocal processes: the stationary Gaussian case. Ann. Math. Statist. 41, 1624-1630.

[12] Lifshits, M. (2012). Lectures on Gaussian processes. SpringerBriefs in Mathematics, Springer, Berlin.

[13] Liu, J. V., Zongfu, H. and MaO, H. (2014). Karhunen-Loève expansion for additive Slepian processes. Stat. Probab. Lett., 90, 93-99.

[14] Mörters, P. and Peres, Y. (2010). Brownian motion. Cambridge Series in Statistical and Probabilistic Mathematics 30, Cambridge University Press, Cambridge.

[15] Nikitin, Ya. Yu. and Orsingher, E. (2006). Sharp small ball asymptotics for Slepian and Watson processes in Hilbert norm. (Russian) Zap. Nauchn. Semin. POMI, 320, 120-128 (2004), translation in J. Math. Sci., New York, 137 No. 1, 4555-4560.

[16] Shepp, L. A. (1966). Radon-Nikodym derivatives of Gaussian measures. Ann. Math. Stat., 37, 321-354.

[17] Shepp, L. A. (1971). First passage time for a particular Gaussian process. Ann. Math. Stat., 42, 946-951.

[18] Slepian, D. (1961). First passage time for a particular Gaussian process. Ann. Math. Stat., 32, 610-612. 